\title{
Psoriasiform Drug Eruption Caused by Abatacept: Immunohistochemical Investigation of STAT Signaling
}

\author{
Kayo Tanita Taku Fujimura Aya Kakizaki Sadanori Furudate \\ Yoshiyuki Kusakari Setsuya Aiba \\ Department of Dermatology, Tohoku University Graduate School of Medicine, Sendai, \\ Japan
}

\section{Key Words}

Psoriasiform drug eruption $\cdot$ pSTAT3 $\cdot$ Interleukin $17 \cdot$ Psoriasis vulgaris

\begin{abstract}
Abatacept is a biological immune modifier that is used for the treatment of rheumatoid arthritis. Although psoriasiform drug eruption is reported as one of the cutaneous adverse effects of abatacept, the precise mechanisms are not fully understood. In this report, we describe a 65 -year-old Japanese man with psoriasiform drug eruption caused by abatacept. Interestingly, immunohistochemical staining revealed that the epidermal keratinocytes in the basal layer and lower layers of the stratum spinosum were positive for PSTAT3, partially positive for PSTAT1 and negative for pSTAT6, which is similar to conventional psoriasis vulgaris. Our present study suggests that psoriasiform drug eruption caused by abatacept might develop by similar immunological mechanisms as those of psoriasis vulgaris.
\end{abstract}

(C) 2015 S. Karger AG, Basel

\section{Introduction}

Abatacept is a biological immune modifier that is used for the treatment of rheumatoid arthritis. Although psoriasiform drug eruption is reported as one of the cutaneous adverse effects of abatacept [1-3], the precise mechanisms are not fully understood. In this report, we describe a case of psoriasiform drug eruption caused by abatacept; we employed immunohistochemical (IHC) staining for pSTAT1, pSTAT3 and pSTAT6 to compare the immu-

KARGER 125/s $\quad \begin{aligned} & \text { Taku Fujimura } \\ & \text { Department of Dermatology } \\ & \text { Tohoku University Graduate School of Medicine } \\ & \text { Seiryo-machi 1-1, Aoba-ku, Sendai 980-8574 (Japan) } \\ & \text { E-Mai tfujimura1@mac.com }\end{aligned}$


Tanita et al.: Psoriasiform Drug Eruption Caused by Abatacept: Immunohistochemical Investigation of STAT Signaling

nological background of the psoriasiform eruption with conventional psoriasis vulgaris as previously reported [4].

\section{Case Report}

A 65-year-old Japanese man visited our outpatient clinic with a 4-month history of pruritic erythema on the scalp. His condition had been treated in a private clinic as psoriasis and he had been administered a topical steroid and maxacalcitol, with inadequate effects. He had a 23-year history of rheumatoid arthritis and had been administered abatacept since the previous year. On his initial visit, physical examination revealed multiple, well-demarcated scaly erythema on the scalp, trunk and extremities (fig. 1). A biopsy specimen showed elongated rete ridges, parakeratosis with neutrophils and dilated tortuous vessels in the dermal papillae (fig. 2a). As we suspected this patient to have psoriasiform dermatitis caused by abatacept, we postponed the administration of abatacept, and the eruption gradually improved by topical steroid and maxacalcitol. Then, we re-administered abatacept, resulting in recurrence of the skin symptoms. From the above findings, we diagnosed the patient as having psoriasiform dermatitis, possibly caused by abatacept.

To further investigate the possible immunological background of the psoriasiform dermatitis by abatacept, we employed IHC staining for pSTAT1 (fig. 2b), pSTAT3 (fig. 2c) and pSTAT6 (fig. 2d). IHC staining revealed that the epidermal keratinocytes in the basal layer and lower layers of the stratum spinosum were positive for pSTAT3, partially positive for pSTAT1 and negative for pSTAT6. As a previous report suggested [4], these data suggested that keratinocytes in the skin express pSTAT1 by the Th1 cytokine IFN- $\gamma$, and pSTAT3 by IL-6, causing Th17 differentiation. Indeed, IHC staining for IFN- $\gamma$ (fig. 2e) and IL-17 (fig. 2f) revealed that both IFN- $\gamma$-producing cells and IL-17-producing cells were prominent in the dermal papule.

\section{Discussion}

Psoriasis is a cutaneous disorder characterized by epidermal hyperproliferation and inflammation. Recently, several studies suggested that pro-inflammatory cytokines, such as IL-17, TNF- $\alpha$ and IL-23, play a crucial role in the pathogenesis of psoriasis, and recombinant monoclonal antibodies for these cytokines have been administered clinically [5, 6]. Among them, Langley et al. [7] reported that a high dose of secukinumab, a fully human anti-IL-17A monoclonal antibody, improves severe eruptions in patients with psoriasis. These reports clearly suggested that these cytokines contribute to the pathogenesis of psoriasis, concurring with another report which suggested that epidermal keratinocytes in psoriasis were positive for pSTAT3, partially positive for pSTAT1 and negative for pSTAT6, suggesting that keratinocytes in the skin express pSTAT1 by the Th1 cytokine IFN- $\gamma$, and pSTAT3 by IL-6, causing Th17 differentiation [4]. These reports suggested that both IL-17 and IFN- $\gamma$ play a role in the development of psoriasis.

Abatacept is a fusion protein of cytotoxic T-lymphocyte-associated protein 4 and human immunoglobulin that blocks the co-stimulation of $\mathrm{T}$ cells, causing downregulation of $\mathrm{T}$ cell activities [1]. Previously, several cases of paradoxical psoriasis during treatment with abatacept have been reported [1-3]. Among them, Konsta et al. [1] reported that 17 of 3,277 RA patients $(0.5 \%)$ who received abatacept developed psoriasis as an adverse event. Since psoriasiform dermatitis is reported as one of the minor side effects of abatacept, the precise 
Tanita et al.: Psoriasiform Drug Eruption Caused by Abatacept: Immunohistochemical Investigation of STAT Signaling

mechanisms for this adverse event are still under discussion. Notably, Bouguermouh et al. [8] reported that B7 co-stimulatory molecules-mediated CD28 signaling in CD4+ naïve $\mathrm{T}$ cells downregulates the induction of Th17, which suggested the possibility that blocking CD28 increases the differentiation of Th17. Indeed, in our present case, the expression of nuclear pSTAT3 in epidermal keratinocytes was prominent, suggesting Th17 differentiation in the dermal papule. Our present study suggests one of the possible mechanisms of the psoriasiform drug eruption caused by abatacept.

\section{Statement of Ethics}

The authors have no ethical conflicts to disclose. The patient provided written informed consent.

\section{Disclosure Statement}

The authors have no conflict of interest to declare.

\section{References}

-1 Konsta M, Rallis E, Karameris A, Stratigos A, Sfikakis PP, Iliopoulos A: Psoriasiform lesions appearing in three patients with rheumatoid arthritis during therapeutic administration of abatacept, a selective inhibitor of T-cell costimulation. J Eur Acad Dermatol Venereol 2012;26:257-258.

-2 Conde-Montero E, Baniandrés-Rodríguez O, Mendoza-Cembranos MD, Horcajada-Reales C, SuárezFernández R: Psoriasiform reactions during treatment with abatacept. Indian J Dermatol Venereol Leprol 2014;80:92-93.

3 Kato K, Satoh T, Nishizawa A, Yokozeki H: Psoriasiform drug eruption due to abatacept. Acta Derm Venereol 2011;91:362-363.

4 Fukushi S, Yamasaki K, Aiba S: Nuclear localization of activated STAT6 and STAT3 in epidermis of prurigo nodularis. Br J Dermatol 2011;165:990-996.

5 Lynde CW, Poulin Y, Vender R, Bourcier M, Khalil S: Interleukin 17A: toward a new understanding of psoriasis pathogenesis. J Am Acad Dermatol 2014;71:141-150.

6 Sorenson E, Koo J: Evidence-based adverse effects of biologic agents in the treatment of moderate-to-severe psoriasis: providing clarity to an opaque topic. J Dermatolog Treat 2015, Epub ahead of print.

7 Langley RG, Elewski BE, Lebwohl M, Reich K, Griffiths CE, Papp K, Puig L, Nakagawa H, Spelman L, Sigurgeirsson B, Rivas E, Tsai TF, Wasel N, Tyring S, Salko T, Hampele I, Notter M, Karpov A, Helou S, Papavassilis C; ERASURE Study Group; FIXTURE Study Group: Secukinumab in plaque psoriasis - results of two phase 3 trials. N Engl J Med 2014;371:326-338.

8 Bouguermouh S, Fortin G, Baba N, Rubio M, Sarfati M: CD28 co-stimulation down regulates Th17 development. PLoS One 2009;4:e5087. 


\section{Case Reports in Dermatology}

Tanita et al.: Psoriasiform Drug Eruption Caused by Abatacept: Immunohistochemical Investigation of STAT Signaling

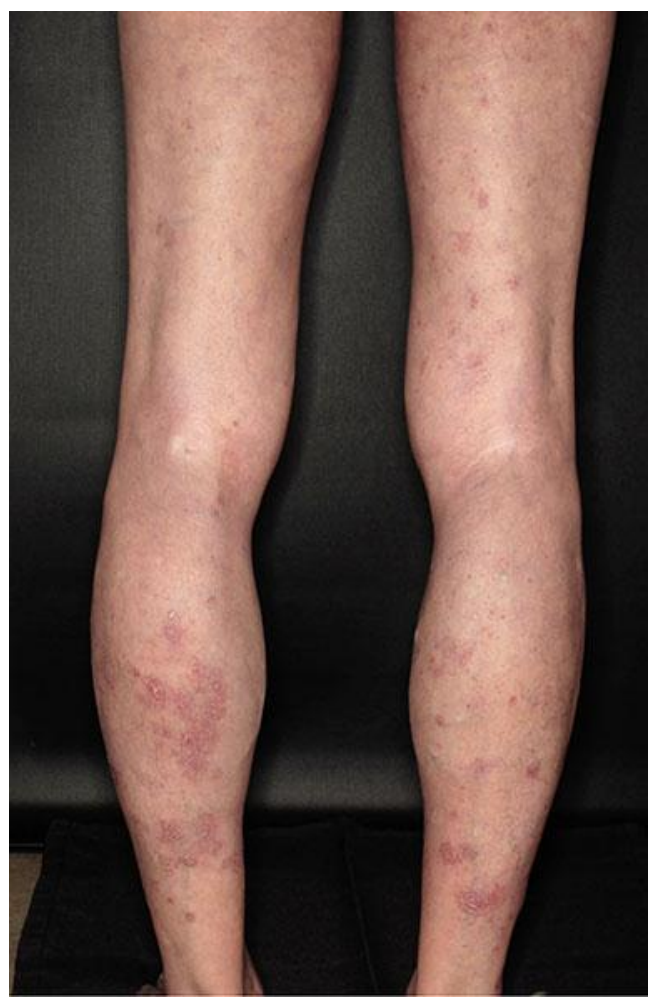

Fig. 1. Multiple, well-demarcated scaly erythema on the extremities. 
Tanita et al.: Psoriasiform Drug Eruption Caused by Abatacept: Immunohistochemical Investigation of STAT Signaling
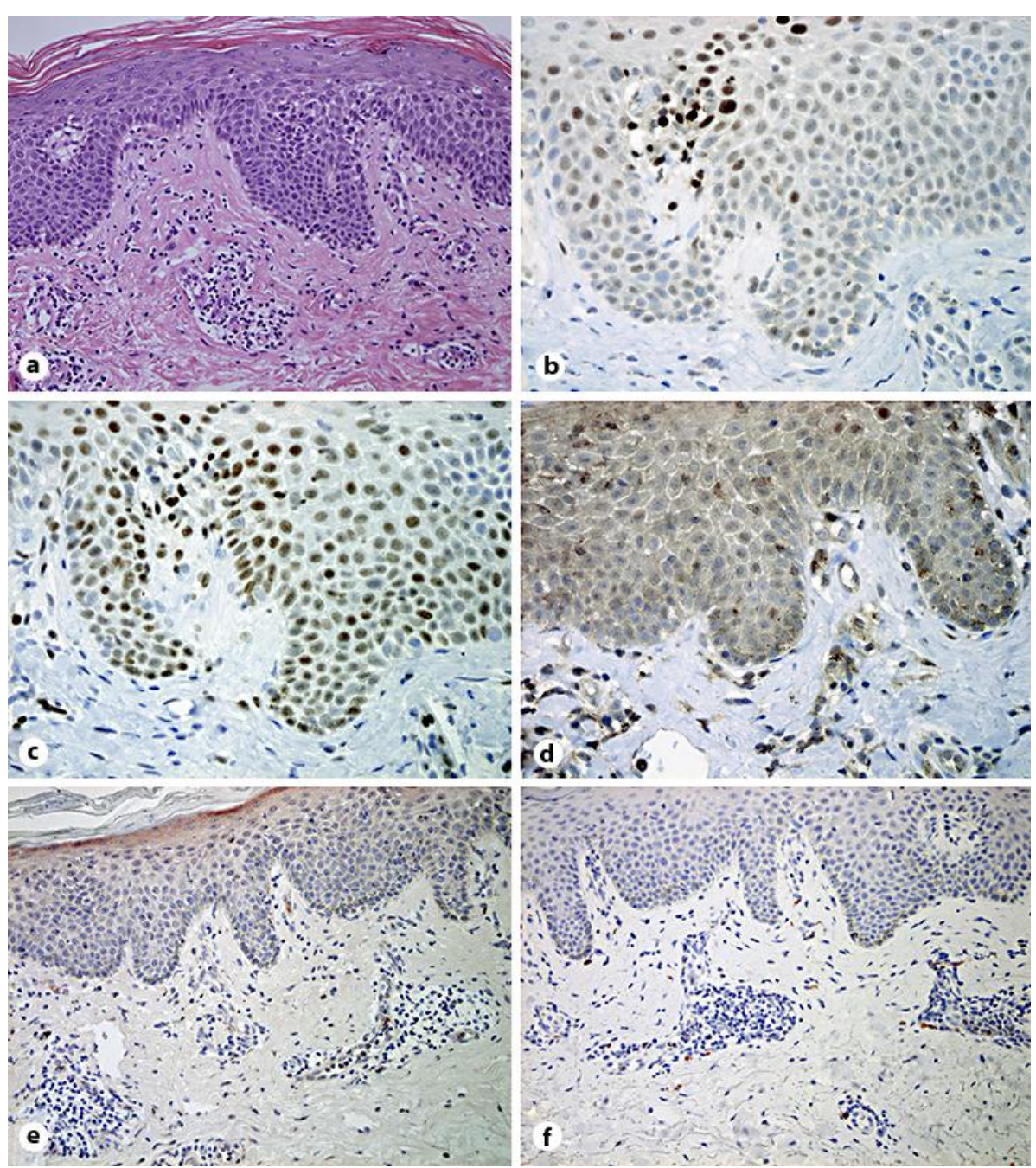

Fig. 2. a Elongated rete ridges, parakeratosis with neutrophils and dilated tortuous vessels in the dermal papillae. b-f Paraffin-embedded tissue samples from the right shoulder were deparaffinized and stained with anti-pSTAT1 antibody (b), anti-pSTAT3 antibody (c), anti-pSTAT6 antibody (d), anti-IFN- $\gamma$ antibody (e) or anti-IL-17 antibody (f). The sections were developed with 3,3'-diaminobenzidine tetrahydrochloride and its enhancer (b-d) or with liquid permanent red $(\mathbf{a}, \mathbf{e}, \mathbf{f})$. Original magnification $\times 200(a, e, f)$ and $\times 400(b-d)$. 